\title{
A GENERIC RIGOROUS SENSOR MODEL FOR PHOTOGRAMMETRIC PROCESSING OF PUSHBROOM PLANETARY IMAGES
}

\author{
Xun Geng ${ }^{1,2, *}$, Shuai Xing ${ }^{1}$, Qing $\mathrm{Xu}^{1}$ \\ ${ }^{1}$ Dept. of Photogrammetry, Zhengzhou Institute of Surveying and Mapping, Zhengzhou 450052, China - (gengxun_rs, \\ xing972403)@163.com,xq@ szdcec.com \\ ${ }^{2}$ Xi'an Information Technique Institute of Surveying and Mapping, Xi'an 710054, China
}

Commission III, ICWG III/II

KEY WORDS: Planetary photogrammetry, Mars mapping, Lunar mapping, Rigorous sensor model, Linear pushbroom images, Orthorectification

\begin{abstract}
:
Currently, each planetary exploration mission team always develops its own software modules to support the photogrammetric processing of planetary images, and as a result of that the main drawbacks are lacking software reusability and the high cost of software development and maintenance. This is mainly due to that there is lack of a highly universal sensor model in the planetary mapping community. This paper presents a generic rigorous sensor model (RSM) for the photogrammetric processing of pushbroom planetary images. The main contributions of this paper include: (1) the implementation details of the generic RSM; (2) the optimized coordinates transformation methods between 3D ground points and 2D image points for linear pushbroom images; (3) a pipeline to acquire exterior orientation (EO) parameters for each planetary image. The generic RSM is developed based on the methodology used in airborne linear scanners ADS40. Specifically, the generic RSM comprises of a camera file and an orientation data file for each image. The camera file stores each detector's calibrated image coordinates and the orientation data file contains each scan line's EO parameters, such that the RSM can perform coordinates transformation among pixel coordinates, focal plane coordinates and ground coordinates. Furthermore, the generic RSM supports varying exposure time, summing mode and image distortions, which are typical problems that need to be solved in planetary mapping. We tested the generic RSM with Lunar Reconnaissance Orbiter (LRO) Narrow Angle Camera (NAC), Chandrayaan-1 Moon Mineralogy Mapper (M3) and Mars Express (MEX) High Resolution Stereo Camera (HRSC) images. The geometric accuracy and computational efficiency of the developed generic RSM were compared with the famous planetary mapping software, namely Integrated System for Imagers and Spectrometers (ISIS). The experimental results demonstrate that the generic RSM has the merits of processing various types of pushbroom planetary images with a unified way and decreasing the software development and maintenance burden. Moreover, the developed generic RSM significantly improves the computational efficiency of orthophoto generation and tie points extraction for pushbroom planetary images.
\end{abstract}

\section{INTRODUCTION}

\subsection{Background}

Linear cameras can provide higher resolution images and wider fields of view than frame cameras at a lower cost. Thus, most planetary exploration imaging instruments for mapping purpose adopt linear cameras (Albertz et al., 2005; Kirk et al., 2008; Di et al., 2014; Speyerer et al., 2016). However, the photogrammetric processing of linear pushbroom images is more complicated than that of frame images. Moreover, various types of linear cameras are designed with different requirements to achieve their respective scientific objectives. Basically, each planetary exploration mission team always develops its own photogrammetric software modules to derive mapping products. Obviously, this lacks software reusability and increases the cost of software development and maintenance.

It is noted that there is lack of a highly universal sensor model in the field of planetary mapping. As the researchers point out, making geometric camera models available in various software packages is still a technical challenging task (Kirk et al., 2012). Furthermore, photogrammetric processing algorithms and software tools always lag behind imaging instrument development. However, if there is a universal sensor model available, these problems can be avoided or minimized as much as possible. Therefore, developing a generic rigorous sensor model (RSM) for processing pushbroom planetary images is very meaningful.

\subsection{Existing Pushbroom Sensor Model}

The most widely used open source planetary mapping software is Integrated Software for Imagers and Spectrometers (ISIS), which was developed by the United States Geological Survey (USGS) Astrogeology Team (Edmundson et al., 2012). ISIS supports many planetary imaging instruments such as Lunar Reconnaissance Orbiter (LRO) Narrow Angle Camera (NAC) and Mars Express (MEX) High Resolution Stereo Camera (HRSC). Recently, the open source automated stereogrammetry software-Ames Stereo Pipeline (ASP), developed by the National Aeronautics and Space Administration (NASA) Ames Research Center, is gradually recognized in planetary mapping community (Shean et al., 2016; Beyer et al., 2018; Tao et al, 2018). ASP has advantages in image matching and digital terrain model (DTM) generation. The authors of ISIS developed

\footnotetext{
* Corresponding author
} 
corresponding sensor model for pushbroom planetary images, and the sensor model parameters are stored in the ISIS cube images. ASP also uses the ISIS sensor model to process planetary images. However, it is noted that the computational efficiency of the ISIS pushbroom sensor model is unsatisfactory, especially in ground-to-image transformation algorithm (Geng et al., 2018). Additionally, to apply more powerful photogrammetric features, commercial photogrammetric workstation such as BAE Systems SOCET SET is always adopted (Kirk et al., 2008; Kirk et al., 2017). Thus, USGS ports the ISIS pushbroom sensor model to the pushbroom sensor model of SOCET SET to conduct practical mapping projects.

\subsection{Our Work}

This paper presents a generic RSM for photogrammetric processing of pushbroom planetary images, and investigates the applications of the developed generic RSM in orthophotos generation and automatic tie points extraction.

\section{GENERIC RIGOROUS SENSOR MODEL FOR PUSHBROOM PLANETARY IMAGES}

\subsection{Generic Rigorous Sensor Model}

Sensor model is mainly used to conduct coordinates transformation between three-dimensional (3D) ground coordinates and two-dimensional (2D) image coordinates, which is the true heart of photogrammetry. For linear pushbroom images, each scan line has its own exterior orientation (EO) parameters, and the central perspective projection only applies in the across-track direction of each scan line. This paper aims at developing a generic RSM that can process various types of pushbroom planetary images in a unified and efficient way. According to the practical processing requirements, the generic RSM should have the following characteristics:

1. supporting multiple planetary bodies such as Moon and Mars;

2. $\quad$ supporting various types of linear cameras, including multi-line and single line scanners;

3. efficient ground-to-image transformation algorithm;

4. be able to solve some practical problems such as summing mode and varying exposure time of scan line;

5. easy to support the error propagation and refinement of the EO parameters.

Based on our work experiences with airborne linear cameras ADS40 (or ADS80/100), the ADS40 sensor model provides a very good foundation for developing the generic RSM relative to pushbroom planetary images. The design principle of the ADS40 sensor model makes it meet most of the characteristics listed above. Specifically, the ADS40 sensor model uses a separate camera file (i.e., .cam) to store each detector's focal plane coordinates. This provides a generalized approach to perform coordinates transformation between pixel coordinates and focal plane coordinates, and avoids considering the detailed image distortions relative to specific imaging instrument. The ADS40 sensor model also uses a separate orientation data file (i.e., .odf) to store each scan line's EO parameters. Thus, it is helpful to solve the problem of varying exposure time within an image. Furthermore, the ADS40 sensor model is easy to support different versions of EO parameters such as the initial EO parameters derived from Spacecraft Planetary Instrument Cmatrix Events (SPICE) kernels or the refined EO parameters. EO parameters of different versions can facilitate the photogrammetric processing in practical mapping projects. In case of error propagation, the orientation data file contains the uncertainties of EO parameters, which can be used to determine the weight values in bundle adjustment. Last but not least, the ADS40 sensor model is well supported by mainstream commercial photogrammetric workstation such as Hexagon Geospatial's IMAGINE Photogrammetry (formerly Leica Photogrammetry Suite-LPS). Therefore, we can make use of more commercial photogrammetric software to process planetary images.

\subsection{Implementation Details}

Figure 1 presents the construction of the generic RSM and the overall photogrammetric processing procedures based on approximate orthophotos.

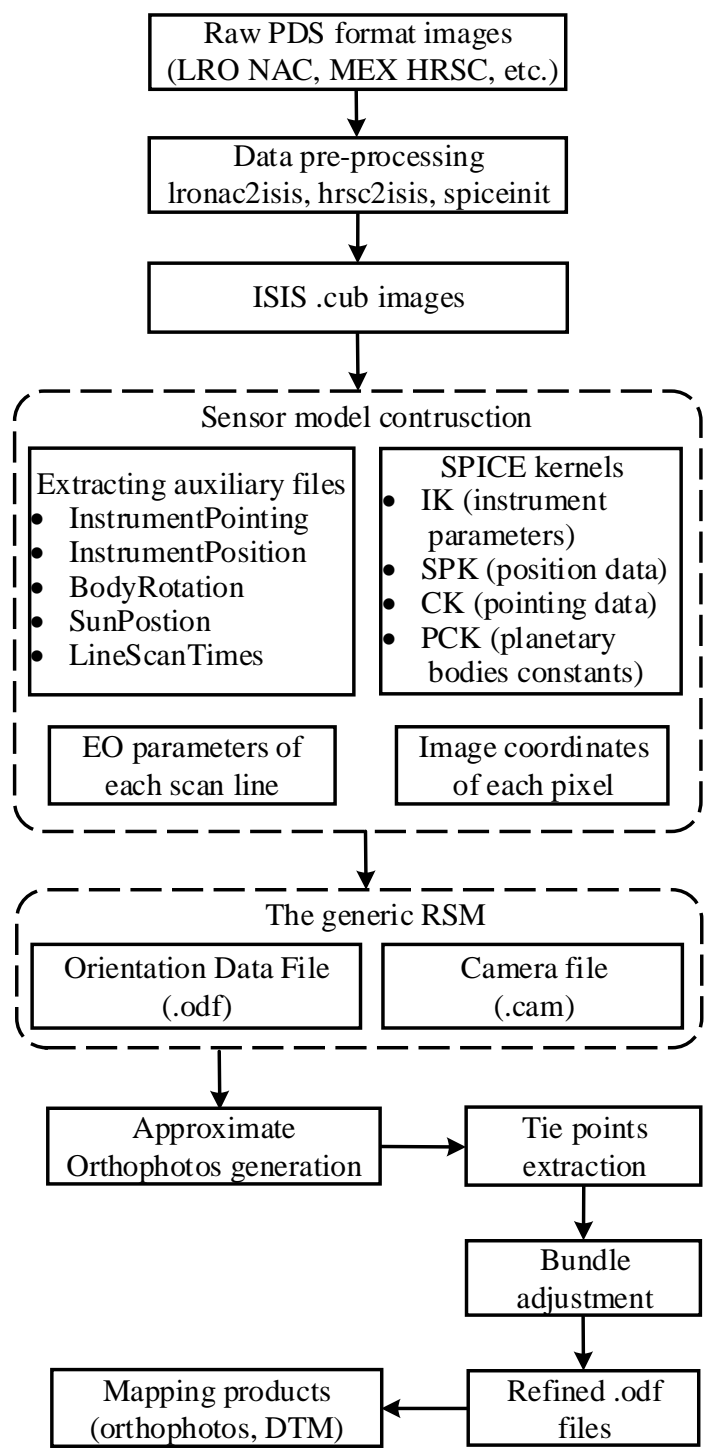

Figure 1. The construction of the developed generic rigorous sensor model (RSM) and the overall photogrammetric processing procedures based on approximate orthophotos.

The detailed calculation steps are as follows:

1. The Planetary Data System (PDS) format raw images are imported into ISIS and converted to ISIS cube (i.e., .cub) images. 
2. The ISIS cube images are initialized using spiceinit, and the ISIS pushbroom sensor model is constructed.

3. Each scan line's EO parameters and each detector's image coordinates are acquired from the ISIS pushbroom sensor model. This step requires some auxiliary position and pointing data files as well as the SPICE kernels.

4. The orientation data file and camera file are generated according to the file format of ADS40 sensor model, respectively.

5. With the constructed generic RSM, approximate orthophotos are generated firstly using the initial orientation data files. Next, tie points are extracted on the approximate orthophotos, and converted to the raw images for bundle adjustment.

6. The refined orientation data files are used to generate high accuracy mapping products (e.g., orthophotos and DTM).

2.2.1 Camera File: The camera file contains the camera geometric parameters such as focal length, pixel size, number of detectors and each detector's calibrated image coordinates. Additionally, the linear array information is stored in the camera file as well. Indeed, the camera file is relative to the linear array instead of the linear camera. Specifically, in case of LRO NAC, there are two camera files for NAC_LEFT and NAC_RIGHT, respectively.

2.2.2 Orientation Data File: The generation of orientation data file requires some auxiliary files, as shown in Table 1 . These auxiliary files can be acquired using ISIS's tabledump. The ADS40 orientation data file format adopts a compression strategy to save storage space. Thus, the orientation data file for a typical LRO NAC image is about $2 \mathrm{MB}$. It is noteworthy that ISIS (e.g., ISIS 3.5.2) implemented the conversion from ISIS pushbroom sensor model to SOCET SET pushbroom sensor model, which facilitates our software development.

\begin{tabular}{|l|c|}
\hline Auxiliary Files & Purpose \\
\hline InstrumentPointing & Acquire the pointing data \\
InstrumentPosition & Acquire the position data \\
BodyRotation & Conduct coordinates transformation \\
SunPosition & Conduct coordinates transformation \\
LineScanTimes & Calculating exact exposure time \\
\hline
\end{tabular}

Table 1. Auxiliary files used to acquire the exterior orientation parameters of pushbroom planetary images.

2.2.3 Ground-to-image Transformation: The ground-toimage transformation is essential to linear pushbroom images. Compared with ISIS pushbroom sensor model, the developed generic RSM adopts a more efficient ground-to-image transformation algorithm, which utilizes the geometric constraints of the central perspective plane (CPP) of the scan line (Wang et al., 2009). In principle, the CPP-based ground-to-image transformation algorithm uses very simple analytic geometry computations to replace the complicated collinearity equation calculations, such that it greatly improves the computational efficiency. It is noteworthy that image distortions also influence the ground-to-image transformation for linear pushbroom images. Therefore, the original CPP-based ground-to-image transformation algorithm was further improved. In short, the linear array with distortions is segmented into multiple line segments, and the exact CPP is determined using simple analytical geometric calculations (Geng et al., 2018).

\subsection{Solving Some Typical Problems}

2.3.1 Summing Mode: The summing mode images are generated by averaging blocks of pixels into "macropixels", and the macropixels value is usually $2,4,8$, and etc. Most pushbroom sensor models in existing photogrammetric software fail to support summing mode. Under this circumstance, one alternative method is enlarging the summing mode images to the full image size before conducting processing. In the developed generic RSM, the problem of summing mode has been fully taken into account. We write the summing mode information to the orientation data file. For example, if the macropixels value is 2 , then we output a key value pair "summingmode $=2$ " in the comment fields of the orientation data file. Thus, when the orientation data file is imported, the summing mode information is interpreted. The pixel coordinates transformation from the summing mode image to the raw detectors of linear array can be written as:

$$
\left\{\begin{array}{l}
d s=(p s-1.0) \times s x+s s \\
d l=(p l-1.0) \times s y+s l
\end{array}\right.
$$

where $(d s, d l)$ indicate the pixel coordinates on the raw detectors of linear array, $(p s, p l)$ indicate the pixel coordinates on the summing mode image, $s x$ and $s y$ indicate the values of macropixels in the along-track and across-track directions respectively, and $(s s, s l)$ indicate the starting sample and starting line of the summing mode image. The inverse transformation, namely from the raw detectors of linear array to the summing mode image, can be written as:

$$
\left\{\begin{array}{c}
p s=(d s-s s) / s x+1.0 \\
p l=(d l-s l) / s y+1.0
\end{array}\right.
$$

2.3.2 Varying Exposure Time: Varying exposure time of scan line is not as common as summing mode in pushbroom planetary images, but it is typical in MEX HRSC images. To accommodate changes of altitude and velocity in the highly elliptical orbit of MEX spacecraft, HRSC always changes the exposure time every few hundred scan lines. However, many photogrammetric software only supports pushbroom images acquired with constant exposure time (Kirk et al., 2017). The key to solve the problem of varying exposure time is to accurately determine the exposure time (ET) of each scan line. Firstly, we should divide the scan lines of an HRSC image into multiple small segments, and each segment has constant line exposure duration (LED). Note that the LED varies with different segments. Then, given a scan line $l$, the exact segment $S$ that contains scan line $l$ can be determined by traversing. Next, the ET relative to scan line $l$ can be calculated by

$$
E T=\operatorname{startLine} E T+(l-\text { startLine }) \times L E D
$$

where startLine indicates the start line of the segment $S$, startLineET indicates the exposure time of the startLine. With the exact ET of the scan line $l$, the EO parameters can be calculated using SPICE kernels. Once the generic RSM is constructed, there is no need to care about the varying exposure time of scan lines in photogrammetric processing procedures. Since we can directly use EO parameters of adjacent scan lines to interpolate the EO parameters of any scan line with subpixel accuracy. This also illustrates the advantage of the developed generic RSM. 


\section{APPLICATIONS OF THE DEVELOPED GENERIC RIGOROUS SENSOR MODEL}

\subsection{Orthophotos Generation}

Orthophotos generation is always based on indirect method. The key to orthophoto generation is determining the corresponding pixel coordinates on the raw image for a pixel on the orthophoto. Thus, the ground-to-image transformation is the most timeconsuming calculation step. The detailed coordinates transformation procedures for orthophotos generation are presented in Figure 2. Here, the generic RSM is applied to conduct a series of coordinates transformation operations.

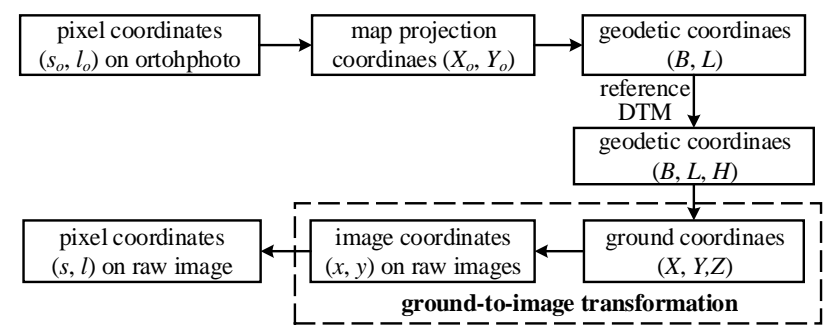

Figure 2. The coordinate transformation procedures in the orthorectification of pushbroom planetary images.

\subsection{Automatic Tie Points Extraction}

Reliable tie points are essential to successful bundle adjustment. It is noted that automatic tie points extraction for pushbroom planetary images, especially for large-scale mapping work, is still a challenging task (Archinal et al., 2012). Recently, researchers tend to conduct image matching of planetary images on orthophotos or approximate orthophotos instead of the original images (Geng et al., 2017; Beyer et al., 2018). The methodology of image matching on orthophotos has advantages of removing image distortions and decreasing the search range of conjugate points. Please note Figure 2 again. It also indicates that we can obtain the corresponding pixel coordinates on the raw image from a pixel on the orthophoto. Therefore, the matched conjugate points on orthophotos can be converted to conjugate points on raw images, and used as tie points for bundle adjustment.

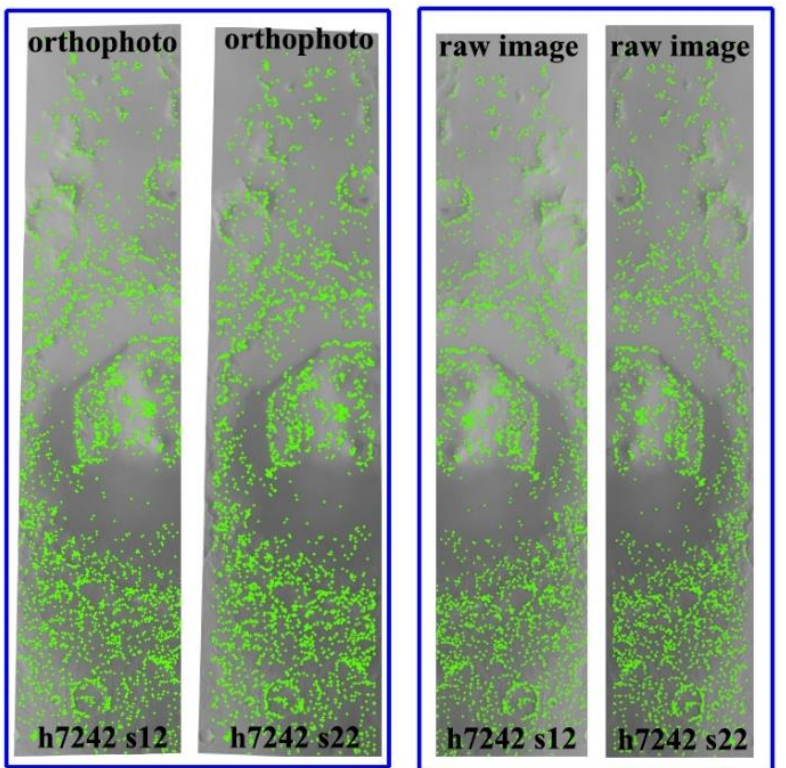

Figure 3. Tie points extraction on orthophotos and converted to the raw images. Green spots indicate conjugate points.
To further illustrate the methodology of image matching on orthophotos, we conduct a tie points extraction test using orthophotos derived from MEX HRSC images. The hardware configuration for this test is shown in section 4 . The classic normalized cross-correlation (NCC) matching method is used. The search window size is $31 \times 31$, the matching window size is $15 \times 15$, and the NCC threshold value is 0.75 . It took 35.9 seconds to complete the image matching for the initial 8610 feature points, and finally 3595 conjugate points were matched (see Figure 3 ). Then, it took only 0.303 seconds to convert these conjugate points on orthophotos to conjugate points on raw images. Note that this conversion time includes the reading and writing of the feature points files.

\section{RESULTS AND DISCUSSION}

The generic RSM was implemented based on the open source photogrammetric software-Dirk's General Analytical Plotter (DGAP, 2019). The software modules for orthophotos generation and tie points extraction were developed using Qt 5.2.1 in the Ubuntu 14.04 operating system installed on a virtual machine. The ISIS 3.5.2 was used to perform pre-processing of planetary images and also used for algorithm comparison. The hardware configurations were an Intel Core i7-7500U with a $2.70 \mathrm{GHz}$ CPU and 8 GB RAM. Though the virtual machine decreased the computational performance to some extent, we can still conduct a fair comparison. Since both ISIS and the developed software run on the same hardware environment. The radius of the reference sphere is $1737.4 \mathrm{~km}$ for lunar images and $3396.19 \mathrm{~km}$ for Martian images, respectively. The test images include LRO NAC, MEX HRSC and Chandrayaan-1 Moon Mineralogy Mapper (M3) images, as listed in Table 2. We firstly examine the geometric accuracy of the generic RSM, followed by evaluation of the orthophotos generation and bundle adjustment results.

\begin{tabular}{|c|c|c|c|c|}
\hline Camera & Images & Date & $\begin{array}{c}\text { GSD } \\
(\mathrm{m})\end{array}$ & $\begin{array}{c}\text { Image } \\
\text { size }\end{array}$ \\
\hline & M109215691LE & 2009.10 .03 & 0.50 & $5064 \times 52224$ \\
& M109215691RE & 2009.10 .03 & 0.50 & $5064 \times 52224$ \\
LRO & M111578606LE & 2009.10 .30 & 0.52 & $5064 \times 52224$ \\
NAC & M111578606RE & 2009.10 .30 & 0.52 & $5064 \times 52224$ \\
& M117467833LE & 2010.01 .07 & 0.42 & $5064 \times 52224$ \\
& M117467833RE & 2010.01 .07 & 0.42 & $5064 \times 52224$ \\
& h5273 nd2 & 2008.02 .09 & 23.63 & $5176 \times 18712$ \\
& h5273 s12 & 2008.02 .09 & 23.19 & $5176 \times 18544$ \\
MEX & h5273 s22 & 2008.02 .09 & 28.08 & $5176 \times 19192$ \\
HRSC & h7242 nd2 & 2009.12 .11 & 16.15 & $5176 \times 24880$ \\
& h7242 s12 & 2009.12 .11 & 16.51 & $5176 \times 24440$ \\
& h7242 s22 & 2009.12 .11 & 18.08 & $5176 \times 25624$ \\
Chan1 & M3G 084125 & 2009.04 .16 & 100.95 & $304 \times 9967$ \\
M3 & M3G 230815 & 2009.06 .09 & 212.78 & $304 \times 11774$ \\
\hline
\end{tabular}

Table 2. Basic information of the test images. GSD refers to ground sample distance. Chan1 indicates Chandrayaan-1.

\subsection{Geometric Accuracy of the Generic Rigorous Sensor Model}

We used three points on one LRO NAC image (i.e., M109215691RE) to perform coordinates transformation among the pixel coordinates (Sample, Line), image coordinates $(x, y)$ and the ground coordinates (Latitude, Longitude, Radius). In case of the image-to-ground transformation, the ground coordinates are 
calculated through projecting the measured pixel coordinates onto a reference DTM. We used the same reference DTM that was used in the ISIS. In case of the ground-to-image transformation, the calculated ground coordinates were backprojected onto the original image to acquire the image coordinates and the corresponding pixel coordinates. Then, the differences between the calculated coordinates and the known ones were calculated, and the results are shown in Table 3. As can be seen, the image coordinates derived with the developed generic RSM were exactly the same as the true values. For ground coordinates, the results show that there were systematic errors of about 8.5E-06 degrees in latitude direction, which was slightly larger than half pixels. This systematic error was mainly introduced by the different interpretation of the first scan line and we will fix it in the future. The residuals in longitude direction were only a few centimeters. It is also noted that the radius direction shows residuals of about tens of centimeters. This was mainly caused by the different interpolation methods that were used at the height value calculation step. Note that the reference DTM for LRO NAC images has a low resolution of $236 \mathrm{~m} / \mathrm{pixel}$, and such that different interpolation methods will yield different height values. From the back-projection columns, it can be observed that the back-projected pixel coordinates were almost the same as the measured pixel coordinates. The residuals were less than 3.1E-06 pixels in the line direction and less than 2.1E08 pixels in the sample direction.

\begin{tabular}{|c|c|c|c|c|c|c|c|c|c|}
\hline \multicolumn{2}{|c|}{ Measured } & \multirow[b]{2}{*}{ Methods } & \multicolumn{2}{|c|}{ Image coordinates } & \multicolumn{3}{|c|}{ Ground coordinates } & \multicolumn{2}{|c|}{ Back-projection } \\
\hline $\begin{array}{c}\text { Sample } \\
\text { (pixel) }\end{array}$ & \begin{tabular}{|c|} 
Line \\
(pixel)
\end{tabular} & & $\begin{array}{c}x \\
(\mathrm{~mm})\end{array}$ & $\begin{array}{c}y \\
(\mathrm{~mm})\end{array}$ & $\begin{array}{l}\text { Latitude } \\
\text { (degree) }\end{array}$ & $\begin{array}{c}\text { Longitude } \\
\text { (degree) }\end{array}$ & $\begin{array}{l}\text { Radius } \\
\text { (meter) }\end{array}$ & $\begin{array}{l}\text { Sample } \\
\text { (pixel) }\end{array}$ & $\begin{array}{c}\text { Line } \\
\text { (pixel) }\end{array}$ \\
\hline \multirow{3}{*}{100} & \multirow{3}{*}{100} & ISIS method & 0 & 16.6861 & 26.52535996 & 3.64580654 & 1735497.2497 & - & - \\
\hline & & Our method & 0 & 16.6861 & 26.52535147 & 3.64580634 & 1735497.2319 & 99.99999998 & 100.00000304 \\
\hline & & Residuals & 0 & 0 & -8.49E-06 & $-2.00 \mathrm{E}-07$ & -0.0178 & $-2.00 \mathrm{E}-08$ & $3.04 \mathrm{E}-06$ \\
\hline \multirow{3}{*}{2500} & \multirow{3}{*}{25000} & ISIS method & 0 & -0.0280 & 26.07678837 & 3.59377080 & 1735390.8419 & - & - \\
\hline & & Our method & 0 & -0.0280 & 26.07677985 & 3.59376993 & 1735391.1212 & 2500.00000001 & 24999.99999794 \\
\hline & & Residuals & 0 & 0 & $-8.52 \mathrm{E}-06$ & $-8.70 \mathrm{E}-07$ & 0.2793 & $1.00 \mathrm{E}-08$ & $-2.06 \mathrm{E}-06$ \\
\hline \multirow{3}{*}{5000} & \multirow{3}{*}{50000} & ISIS method & 0 & -17.4300 & 25.62653583 & 3.53728297 & 1737087.0060 & - & - \\
\hline & & Our method & 0 & -17.4300 & 25.62652766 & 3.53728191 & 1737087.5740 & 5000.00000001 & 49999.99999800 \\
\hline & & Residuals & 0 & 0 & $-8.17 \mathrm{E}-06$ & $-1.06 \mathrm{E}-06$ & 0.5680 & 1.00E-08 & $-2.00 \mathrm{E}-06$ \\
\hline
\end{tabular}

Table 3. Geometric accuracy evaluation of the generic rigorous sensor model using ISIS as reference.

\subsection{Orthophotos Generation Results}

As shown in Figure 4, six images were used to evaluate the orthophotos generation results. The two Chandrayaan-1 M3 images were acquired with summing mode and the macropixels value was 2 . It is noted that the computational performance of this experiment was not as good as that presented in (Geng et al., 2018), due to that virtual machine was used in this test. However, the results still show that the computational efficiency of the developed method is much better than that of ISIS, due to the optimized ground-to-image transformation algorithm.

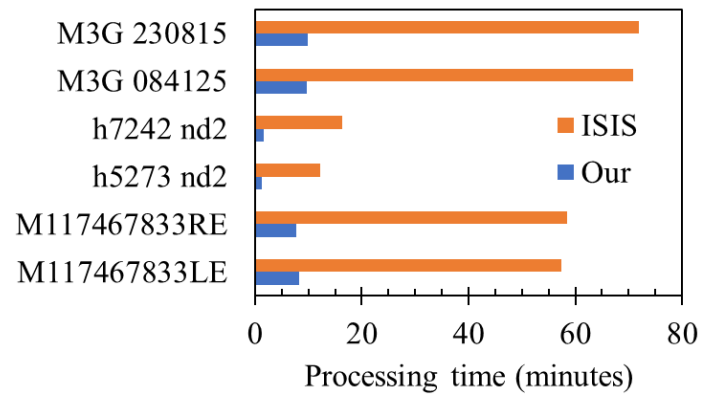

Figure 4. Orthophotos generation experimental results.

\subsection{Bundle Adjustment Results}

The bundle adjustment experiments were conducted using the LRO NAC and MEX HRSC images listed in Table 2. In order to compare and evaluate the tie points extraction results, we also conducted bundle adjustment experiment using tie points generated from ISIS's pointreg. Overall, pointreg is robust and is suitable for various types of planetary images. The main drawback of pointreg is that it conducts image matching on the original image domain, without making use of the geometric information of stereopairs to predict the start position of the conjugate points. Therefore, the search space for pointreg should be relatively large compared with our method. Moreover, we fail to extract enough tie points using pointreg for MEX HRSC images, due to the large differences in imaging angle and varying exposure time. Thus, the comparison test was only performed for LRO NAC images. In case of our method, tie points were automatically extracted using the method introduced in section 3.2. The matched tie points using our method were converted to a Parameter Value Language (PVL) format control network file that can be interpreted by ISIS. The bundle adjustment was conducted with ISIS's jigsaw. Additionally, in ISIS software package the term "control measure" is always used to express tie point, such that we will use both terms in this paper.

4.3.1 Tie Points Extraction: We used ISIS's autoseed to generate initial candidate control measures. Since not all candidate control measures can be matched, a dense tie points grid is used for autoseed. Specifically, the spacing in the $\mathrm{X}$ and $\mathrm{Y}$ directions is $250 \mathrm{~m}$ for LRO NAC test and $9000 \mathrm{~m}$ for MEX HRSC test. Consequently, the number of candidate control measures for LRO NAC and MEX HRSC tests are 3587 and 2850 respectively. In case of our method, the NCC and pyramid matching algorithms were used. The matching window size for both our method and ISIS was $15 \times 15$, whereas the search window size was $31 \times 31$ for our method and $300 \times 300$ for ISIS. Since ISIS requires a large search range to determine the conjugate points. It is well known that the matched conjugate points inevitably contain some outliers, which should be removed before or in the bundle adjustment procedure. We removed the control measures with image coordinates residuals larger than one pixel. The valid control measures for MEX HRSC test are presented in Figure 5. The figure was drawn using ISIS's qnet.

Table 4 lists the number of valid control measures on each image and the corresponding image coordinates residuals. For both 
LRO NAC and MEX HRSC test, most of the image coordinates residuals were less than half pixels in both sample and line directions. It is also observed that the sample residuals of the MEX HRSC test were slightly larger than that of the LRO NAC test. This can be explained by the lower quality of SPICE kernels corresponding to the MEX HRSC images. Table 5 lists the total number of candidate control measures and the corresponding processing time of tie points extraction. Additionally, as can be seen, the Sigma0 values of both LRO NAC and MEX HRSC tests were less than 0.5 , indicating satisfactory bundle adjustment results. From Table 4 and 5, it can be observed that the image coordinates residuals and Sigma0 value of our method were almost the same as that of ISIS. However, our method was more efficient in tie points extraction.

\begin{tabular}{|c|c|c|c|c|c|c|c|}
\hline \multirow{2}{*}{ Camera } & \multirow{2}{*}{ Images } & \multicolumn{2}{|c|}{$\begin{array}{c}\text { Control } \\
\text { measures }\end{array}$} & \multicolumn{2}{c|}{$\begin{array}{c}\text { Sample res. } \\
\text { (pixels) }\end{array}$} & \multicolumn{2}{c|}{$\begin{array}{c}\text { Line res. } \\
\text { (pixels) }\end{array}$} \\
\cline { 3 - 8 } & & Our & ISIS & Our & ISIS & Our & ISIS \\
\hline \multirow{4}{*}{ LRO } & M..691LE & 385 & 154 & 0.09 & 0.11 & 0.48 & 0.53 \\
NAC & M...691RE & 60 & 88 & 0.15 & 0.13 & 0.40 & 0.53 \\
& M..606LE & 398 & 175 & 0.09 & 0.11 & 0.49 & 0.55 \\
& M..833LE & 45 & 83 & 0.17 & 0.15 & 0.32 & 0.53 \\
& M..833RE & 33 & 17 & 0.16 & 0.16 & 0.32 & 0.38 \\
& h5273 nd2 & 88 & - & 0.21 & 0.16 & 0.39 & 0.18 \\
& h5273 s12 & 93 & - & 0.50 & - & 0.40 & - \\
MEX & h5273 s22 & 101 & - & 0.60 & - & 0.41 & - \\
HRSC & h7242 nd2 & 70 & - & 0.29 & - & 0.38 & - \\
& h7242 s12 & 50 & - & 0.54 & - & 0.40 & - \\
& h7242 s22 & 74 & - & 0.45 & - & 0.22 & - \\
\hline
\end{tabular}

Table 4. Valid control measures and image coordinates residuals, where res. indicates residuals.

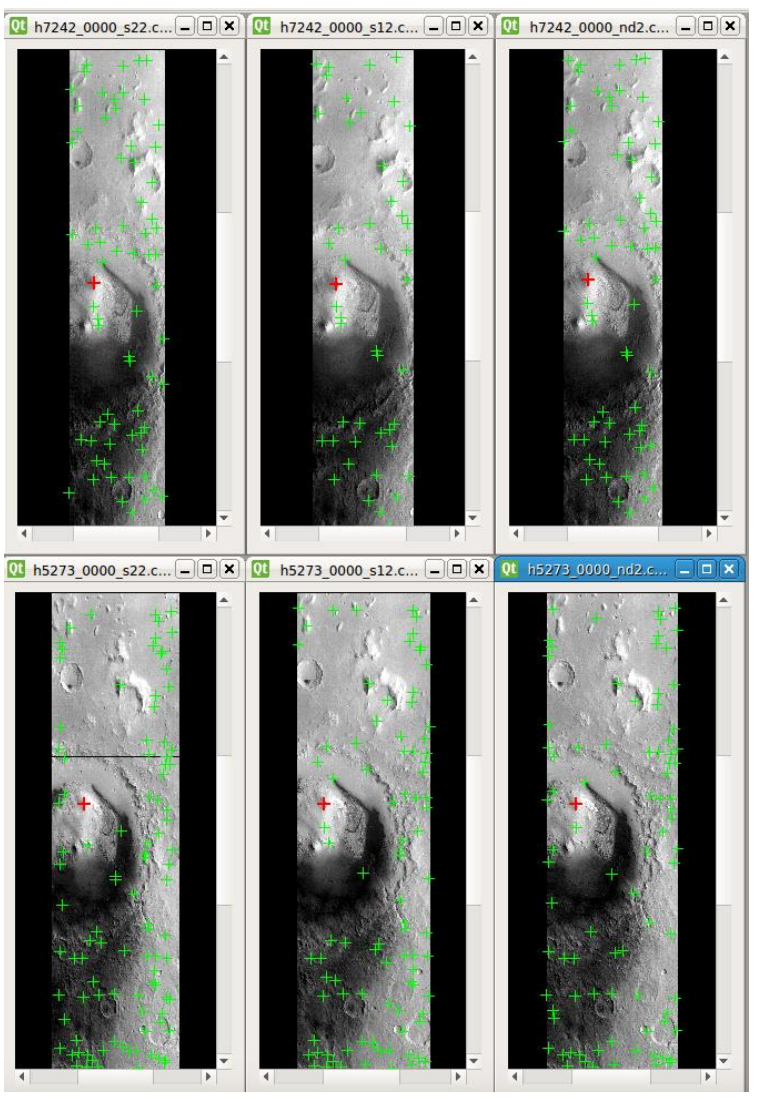

Figure 5. Valid control measures used for bundle adjustment of MEX HRSC images. The black line on h5273 s22 image was introduced at the raw image acquisition period.

\begin{tabular}{|c|c|c|c|c|c|c|}
\hline \multirow{2}{*}{ Camera } & \multicolumn{2}{|c|}{$\begin{array}{c}\text { Candidate } \\
\text { control measures }\end{array}$} & $\begin{array}{c}\text { Processing time } \\
\text { (seconds) }\end{array}$ & \multicolumn{2}{c|}{ Sigma0 } \\
\cline { 2 - 7 } & Our & ISIS & Our & ISIS & Our & ISIS \\
\hline LRO NAC & 3587 & 3587 & 202 & 3540 & 0.45 & 0.43 \\
MEX HRSC & 2850 & - & 220 & - & 0.48 & - \\
\hline
\end{tabular}

Table 5. The overall bundle adjustment results and processing time of tie points extraction.

4.3.2 Geopositioning Accuracy: Figure 6 8 present the geopositioning accuracy of tie points. Here we use $0.5 \mathrm{~m}$ as the average ground sample distance (GSD) of LRO NAC images. Thus, in case of LRO NAC test, the geopositioning accuracy in latitude and longitude directions were about one pixel for our method (see Figure 6), and slightly larger than one pixel for ISIS (see Figure 7). Whereas in the radius direction, it is observed that our method was slightly better than ISIS. Most of the tie points' radius accuracy were about $1.5 \mathrm{~m}$ ( $\sim 3$ pixels) for our method and $2.0 \mathrm{~m}$ ( 4 pixels) for ISIS. For MEX HRSC test, the geopositioning accuracy were only derived from our method, and there was no comparative test result available. As shown in Table 2 , the GSD of MEX HRSC images varies between 16 28 m. We use $20 \mathrm{~m}$ as the average GSD. Figure 8 shows that most of the tie points' geopositioning accuracy were better than $20 \mathrm{~m}$ in latitude and longitude directions, which was equal to about one pixel relative to the average GSD. Similar to the bundle adjustment results of LRO NAC test, the geopositioning accuracy in radius direction was lower than that in the latitude and longitude directions for MEX HRSC test. Overall, both LRO NAC and MEX HRSC tests delivered a satisfying geopositioning accuracy.

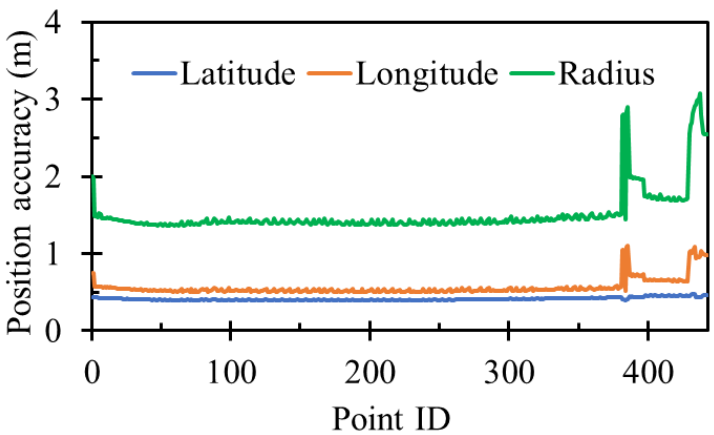

Figure 6. Geopositioning accuracy of tie points for LRO NAC images (our method).

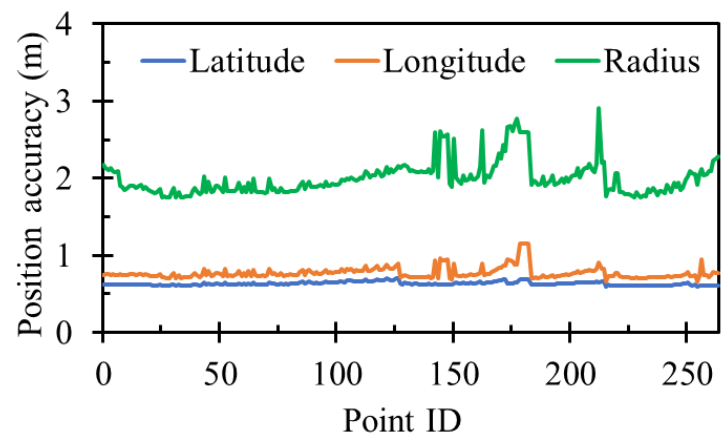

Figure 7. Geopositioning accuracy of tie points for LRO NAC images (ISIS method). 


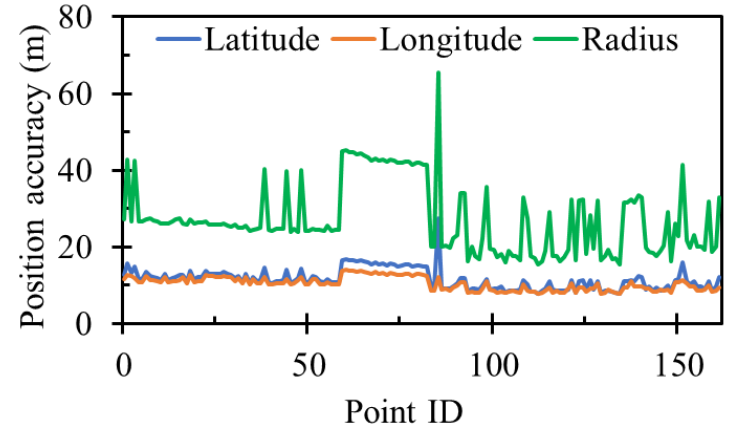

Figure 8. Geopositioning accuracy of tie points for MEX HRSC images (our method).

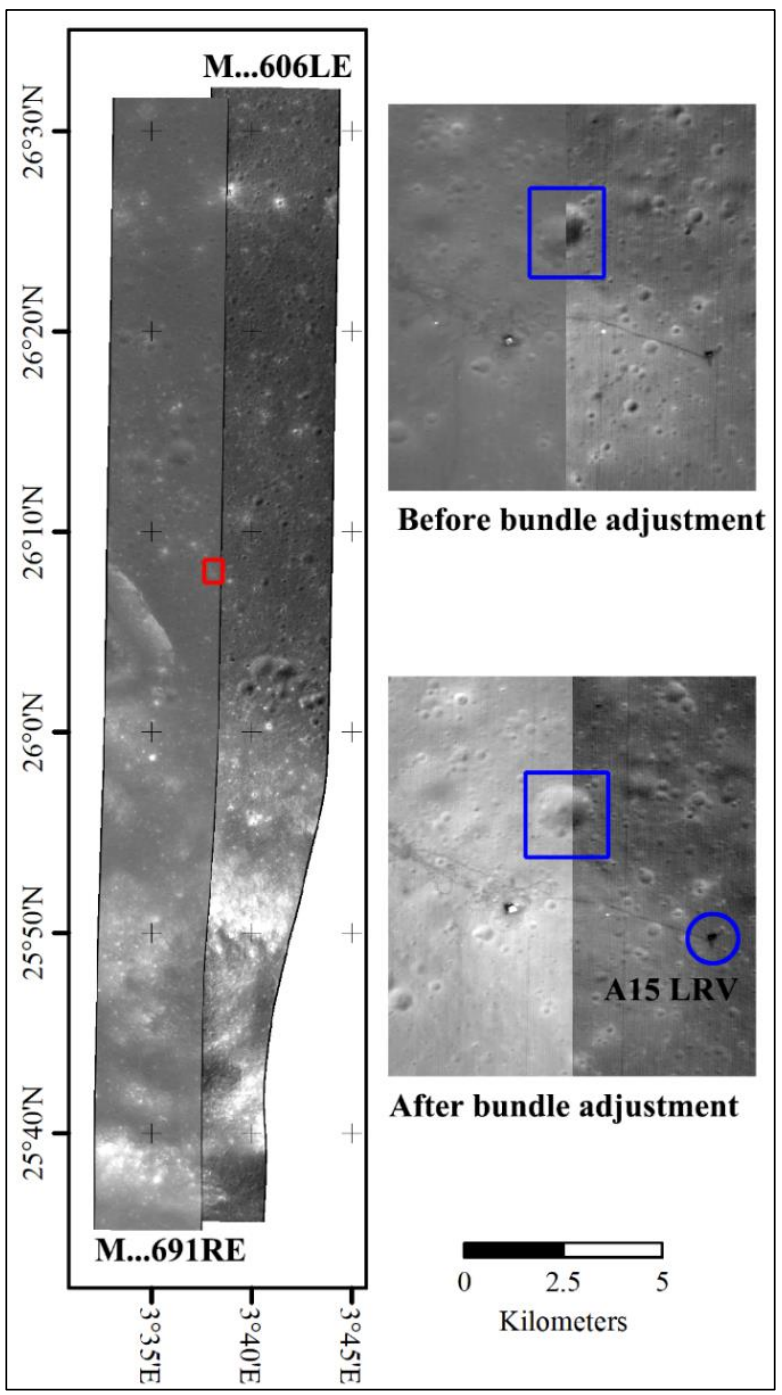

Figure 9. Image mosaics composed of the orthophotos derived from M109215691RE and M111578606LE images. A15 LRV indicates Apollo 15 Lunar Roving Vehicle.

After the bundle adjustment, the ISIS cube images can be updated with the refined EO parameters. Then we can generate orthophotos using these updated cube images. Figure 9 present the mosaic images derived from LRO NAC images. The red rectangle in the middle of the mosaic image indicates the enlarged area in the right parts of the figure. Note the two blue rectangles. It is observed that the bundle adjustment decreased the mosaic displacements from tens of pixels to subpixel. The blue circle in Figure 9 marks the location of Apollo 15 Lunar Roving Vehicle (LRV). Since the accurate coordinates of it is available in (Wagner et al., 2017). Thus, we measured the ground coordinates of Apollo $15 \mathrm{LRV}$ on the generated orthophoto and compared the measured coordinates with the known values. The ground coordinates residuals were $1.85 \mathrm{~m}$ in longitude direction and $-3.16 \mathrm{~m}$ in latitude direction respectively. Since we did not perform absolute orientation, such difference was acceptable. It is noteworthy to point out that the derived geopositioning accuracy was in fact a relative orientation solution. Since we did not use control data in the bundle adjustment procedure. To acquire absolute orientation results, one can manually select some ground control points from reference DTM or adopt some automatic controlling methods (Wu et al., 2014; Xin et al., 2018). However, this paper only presents the relative orientation results to evaluate the developed generic RSM.

\subsection{Discussion}

As shown in Table 3, though the developed generic RSM shows small systematic errors in latitude direction, its internal coincidence accuracy is very high. The experimental results demonstrate the correctness of the developed generic RSM. Based on the optimized ground-to-image transformation algorithm, the computational efficiency of orthophotos generation has been greatly improved. The feasibility of extracting tie points on approximate orthophotos has been verified as well. The methodology of tie points extraction on orthophotos can result in similar bundle adjustment accuracy as ISIS, but delivers a higher computational efficiency. The methodology of image matching on orthophotos also depends on the developed generic RSM to accurately convert pixel coordinates from orthophotos to raw images. The mosaic images results (see Figure 9) show that the bundle adjustment improves the geometric accuracy of planetary images acquired from multiple orbits. This again demonstrates the effectiveness of the developed generic RSM. Furthermore, due to that different types of linear pushbroom images share the same code base, the developed generic RSM greatly ease our software development.

\section{CONCLUSION}

This paper presents a generic RSM for planetary photogrammetry. The airborne ADS40 pushbroom sensor model is extended to develop the generic RSM. The developed generic RSM can process various types of pushbroom planetary images in a unified way, decreasing the software development and maintenance burden. We also investigated the applications of the generic RSM, including orthophotos generation and tie points extraction. Through optimizing the ground-to-image transformation algorithm, orthophotos of pushbroom planetary images can be derived more efficiently. Consequently, the methodology of extracting tie points on orthophotos shows more practical values. It is hoped that the developed generic RSM and the methodology of tie points extraction on orthophotos can be applied to largescale mapping projects involving thousands of planetary images. Obviously, this requires more comprehensive experimental verification. Moreover, to make the proposed method more practical, the orthophoto generation procedure should be further improved for parallel processing.

Though the experimental results show that our method is better than ISIS in some algorithms, the developed software modules are still in demo stage. Currently, we only support three imaging instruments, namely LRO NAC, Chandrayaan-1 M3 and MEX HRSC, and the amount of test images is also limited. Additionally, 
though the implementation of the generic RSM is based on the widely used ADS40 sensor model, there are still many problems in practical use. For example, the summing mode information that stored in the comment fields of the orientation data file cannot be interpreted by existing open source (e.g., DGAP) and commercial software (e.g., LPS).

Indeed, sensor model is the basis of planetary photogrammetry. It is mentioned in the literature that USGS planned to port the optimized ISIS pushbroom sensor model to the Community Sensor Model (CSM) and release the CSM source code under open source license (Kirk et al., 2017). However, it is a pity that we haven't got any more references on CSM for planetary images at present. Moreover, the practical application of a sensor model is affected by many factors. The most important thing is that standards need to be established and adhered to. It is predictable that the CSM relative to planetary images developed by USGS will be available in the near future. Such significant work will greatly facilitate the software development for planetary imaging instruments. Anyway, our work is still meaningful. After all, more options will help to process massive planetary images.

\section{ACKNOWLEDGEMENTS}

This study was supported by the National Natural Science Foundation of China with project number 41401533, 41371436, 41876105. We thank the authors of the open source photogrammetric software (i.e., DGAP and ISIS). We also thank the LRO NAC Team, Chandrayaan-1 M3 team and MEX HRSC team for providing the planetary images to the public.

\section{REFERENCES}

Albertz, J., Attwenger, M., Barrett, J., Casley, S., Dorninger, P., Dorrer, E., Ebner, H., Gehrke, S., Giese, B., Gwinner, K., et al., 2005. HRSC on Mars Express-photogrammetric and cartographic research. Photogramm. Eng. Remote Sens., 71, $1153-1166$.

Archinal, B.A., Kirk, R.L., Keszthelyi, L.P., Gaddis, L.R., Rosiek, M.R., 2012. Can (and will) the data be processed? Technology development to address science questions. International Workshop on Instrumentation for Planetary Missions, 1151.

Beyer, R.A., Alexandrov, O., McMichael, S., 2018. The Ames Stereo Pipeline: NASA's open source software for deriving and processing terrain data. Earth and Space Sci., 5, 537-548.

DGAP, 2019. DGAP, the photogrammetric bundle adjustment software. $<$ http://www.ifp.unistuttgart.de/publications/software/openbundle/index.en.html> (accessed 21-March-2019).

Di, K.C., Liu, Y.L., Liu, B., Peng, M., Hu, W.M., 2014. A selfcalibration bundle adjustment method for photogrammetric processing of Chang'E-2 stereo lunar imagery. IEEE Trans. Geosci. Remote Sens., 52(9), 5432-5442.

Edmundson, K.L., Cook, D.A., Thomas, O.H., Archinal, B.A., Kirk, R.L., 2012. Jigsaw: the ISIS3 bundle adjustment for extraterrestrial photogrammetry. ISPRS Ann. Photogramm. Remote Sens. Spatial Inf. Sci., I-4, 203-208. doi.org/10.5194/isprsannals-I-4-203-2012.
Geng, X., Xu, Q., Xing, S., Lan, C.Z., Xu, J.Y., 2017. A novel pixel-level image matching method for Mars Express HRSC linear pushbroom imagery using approximate orthophotos. Remote Sens., 9, 1262.

Geng, X., Xu, Q., Lan, C.Z., Xing, S., Hou, Y.F., Lyu, L., 2018. Orthorectification of planetary linear pushbroom images based on an improved back projection algorithm. IEEE Geosci. Remote Sens. Lett., in press.

Kirk, R.L., Kraus, E.H., Rosiek, R.M., Anderson, J.A., Archinal, B.A., Becker, K.J., Cook, D.A., Galuszka, D.M., Geissler, P.E., Hare, T.M, et al., 2008. Ultrahigh resolution topographic mapping of Mars with MRO HiRISE stereo images: meter-scale slopes of candidate Phoenix landing sites. J. Geophys. Res. Planet., 113, 5578-5579.

Kirk, R.L., Archinal, B.A., Gaddis, L.R., Rosiek, M.R., 2012. Lunar cartography: progress in the 2000s and prospects for the 2010s. Int. Arch. Photogramm. Remote Sens. Spatial Inf. Sci., XXXIX-B4, 489-494. doi.org/10.5194/isprsarchives-XXXIXB4-489-2012.

Kirk, R.L., Howington-Kraus, E., Edmundson, K., Redding, B., Galuszka, D., Hare, T., Gwinner, K., 2017. Community tools for cartographic and photogrammetric processing of Mars Express HRSC images. Int. Arch. Photogramm. Remote Sens. Spatial Inf. Sci., XLII-W1, 69-76. doi.org/10.5194/isprs-archives-XLII-3W1-69-2017.

Shean, D.E., Alexandrov, O., Moratto, Z.M., Smith, B.E., Joughin, I.R., Porter, C., Morin, P., 2016. An automated, opensource pipeline for mass production of digital elevation models (DEMs) from very-high-resolution commercial stereo satellite imagery. ISPRS J. Photogramm. Remote Sens., 116, 101-117.

Speyerer, E.J., Wagner, R.V., Robinson, M.S., Licht, A., Thomas, P.C., Becker, K., et al., 2016. Pre-flight and on-orbit geometric calibration of the Lunar Reconnaissance Orbiter Camera. Space Sci. Rev., 200, 357-392.

Tao, Y., Muller, J.P., Sidiropoulos, P., Xiong, S.T., Putri, A.R.D., Walter, S.H.G., Veitch-Michaelis, J., and Yershov, V., 2018. Massive stereo-based DTM production for Mars on cloud computers. Planetary Space Sci., 154, 30-58.

Wagner, R.V., Nelson, D.M., Plescia, J.B., Robinson, M.S., Speyerer, E.J., Mazarico, E., 2017. Coordinates of anthropogenic features on the Moon. Icarus, 283, 92-103.

Wang, M., Hu, F., Li, J., Pan, J., 2009. A fast approach to best scanline search of airborne linear pushbroom images. Photogramm. Eng. Remote. Sens., 75, 1059-1067.

Wu, B., Hu, H., Guo, J., 2014. Integration of Chang'E-2 imagery and LRO laser altimeter data with a combined block adjustment for precision lunar topographic modeling. Earth Planet. Sci. Lett., $391,1-15$.

Xin, X., Liu, B., Di, K.C., Jia, M.N., Oberst, J., 2018. Highprecision co-registration of orbiter imagery and digital elevation model constrained by both geometric and photometric information, ISPRS J. Photogramm. Remote Sens., $144,28-37$. 